International Journal of Applied Mathematics

Volume 32 No. $6 \quad 2019,925-932$

ISSN: 1311-1728 (printed version); ISSN: 1314-8060 (on-line version)

doi: http://dx.doi.org/10.12732/ijam.v32i6.2

\title{
A STUDY OF INERTIA INDICES, SIGNATURE AND NULLITY OF V-PHENYLENIC $[m, n]$
}

\author{
Zheng-Qing $\mathrm{Chu}^{1}$, Asim Razzaq ${ }^{2}$, \\ Kashif Ali ${ }^{2}$, Syed Tahir Raza Rizvi ${ }^{2}$ \\ ${ }^{1}$ Department of General Education \\ Anhui Xinhua University \\ Hefei 230088, CHINA \\ ${ }^{2}$ Department of Mathematical Sciences \\ COMSATS Institute of Information Technology \\ Lahore, PAKISTAN
}

\begin{abstract}
A molecular/chemical graph is hydrogen depleted chemical structure in which vertices denote atoms and edges denote the bonds. Topological descriptors are the numerical indices based on the topology of the atoms and their bonds (chemical conformation, quaternary structure). They correlate various physico-chemical properties like boiling point, enthalpy of formation, enthalpy of vaporization, Kovat's constant etc., of various chemical compounds. The numerical parameters like inertia indices, signature and nullity attract much attention due to their diverse application in chemistry, e.g. nullity of a molecular graph is related to the stability of saturated hydrocarbons. In this paper, we determine the inertia indices, signature and nullity of V-phenylenic $[m, n]$ nanotube. We also study these numerical parameters for line graph of V-phenylenic $[m, n]$ nanotube.
\end{abstract}

AMS Subject Classification: 05C07, 05C35, 05C40

Key Words: inertia, singularity, nullity, V-phenylenic

\section{Introduction}

Let $G=(V, E)$ be a finite, simple, planar, connected and undirected graph,

Received: June 17, 2019

(c) 2019 Academic Publications

$\S$ Correspondence author 
where $V$ and $E$ are its vertex-set and edge-set respectively. The line graph of a graph $G$, denoted by $L(G)$, is the graph whose vertex set is $E(G)$ and two vertices of $L(G)$ are adjacent if the corresponding edges of $G$ are incident. Let $G$ be an $n$-vertex molecular graphs with vertex-set $V(G)=\left\{v_{1}, v_{2}, \ldots, v_{n}\right\}$ and edge-set $E(G)$. The vertices of $G$ correspond to atoms and an edge between two vertices correspond the chemical bond between these atoms. Line graphs furnish a tool for studying the topological properties of $\sigma$-systems.

The adjacency matrix $A(G)=\left[a_{i j}\right]_{n \times m}$ (usually denoted by $A$ ) of the graph $G$ is defined as:

$$
a_{i j}=\left\{\begin{array}{rl}
1, & \text { if } v_{i} v_{j} \in E(G) \\
0, & \text { if otherwise }
\end{array},\right.
$$

where, $v_{i}, v_{j} \in V(G)$.

The characteristic polynomial of graph $G$ is a polynomial of degree $n$, defined as $f(G, \lambda)=\operatorname{det}\left(\lambda I_{n}-A\right)$, where $I_{n}$ denotes the identity matrix of order $n$. The zeros of $f(G, \lambda)$ are eigenvalues of $A$ and multi-set of eigenvalues of $A$ is called the spectrum of $A$. The eigenvalues and spectrum of $A$ are respectively called the eigenvalue and spectrum of the graph $G$. Since $G$ is a simple graph, the matrix $A$ will have all real eigenvalue and their sum is zero, [7]. The notations used in this article are mainly taken from book [11].

The positive (respectively negative) inertia index of a graph $G$, denoted by $p(G)$ (respectively $n(G)$ ), is defined to be the number of positive (respectively negative) eigenvalues of its adjacency matrix. The signature of $G$, denoted by $s(G)$, is defined as the difference between positive and negative eigenvalues of $G$. The nullity of $G$, symbolized as $\eta(G)$, is defined as the multiplicity of eigenvalue zero in adjacency spectrum of $G$. Obviously $p(G)+n(G)+\eta(G)=|V(G)|$. Nullity of a chemical graph is related to the stability of saturated hydrocarbon. If $\eta(G)>0$ (resp. $\eta(G)=0$ ), then $\mathrm{G}$ is said to be singular (resp. nonsingular). Therefore, a chemical structure is stable (unstable) if nullity of graph is zero (non-zero). These parameters attract much attention of the researchers in the mathematical, theocratical and computational chemistry due to their direct application in chemistry. For further studies of these parameters in different perspective, $[2,12]$.

Carbon nanotube is allotropes of carbon with a cylindrical nanostrructures. These cylindrical carbon molecules have usual properties which are valuable for nanotechnology, electronics, optics and other field of material sciences and technology. With such a huge industrial applications, carbon nanotubes have attracted many researchers to investigate more properties of these nanomaterials and still many things need to explore in this area. 
Nanotubes are members of the fullerene structure family. A fullerene is a carbon molecule in the shape of a hollow sphere, ellipsoid, tube etc. Those whose shape resemble are sphere are called buckyball and those which are in the form of a tube are called buckytubes or nanotubes. Structure of a nanotube is similar to a graphite sheet. Carbon nanotubes appear in different forms with respect to their length, thickness, number of layers and types of the rings which cover the walls of the nanotubes, [1].

Let $G$ denotes the two dimensional lattice of a V-phenylenic $[m, n]$ pattern. Fig. 1 shows a V-phenylenic $[m, n]$ with $m=4$ and $n=4$ and Fig. 2 shows its line graph.

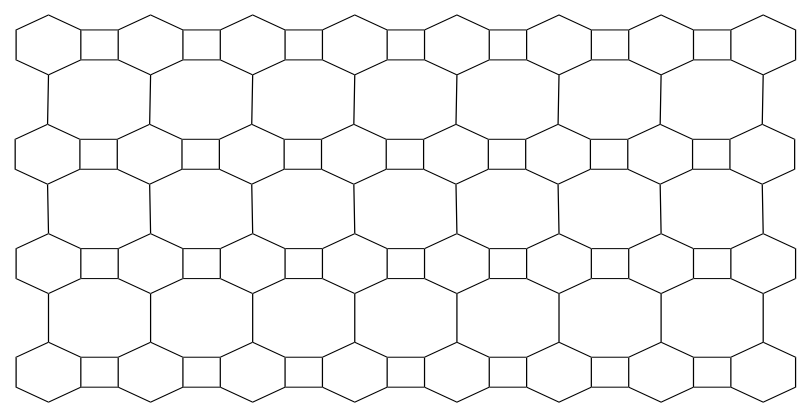

Figure 1: The V-phenylenic $[4,4]$

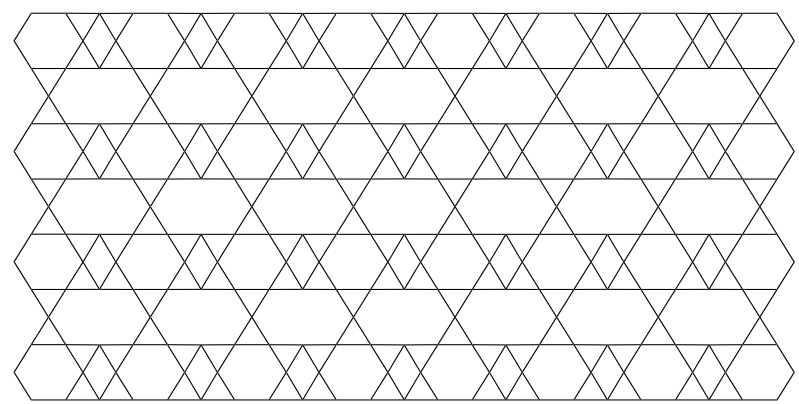

Figure 2: The Line of V-phenylenic $[4,4]$

In this paper, we estimate the inertia indices, signature and nullity of the graph. 


\section{Computational results}

In this section, we explain the procedure to calculate the inertia, signature and nullity for the graph $G$ and its line graph $L(G)$ as follows:

1. The molecules of $G$ and $L(G)$ are drawn in HyperChem [13] for each value of $m$ and $n$ and save the file as .hin type.

2. The adjacency matrices of these molecular graphs are constructed with the help of TopoCluj [8].

3. Then the inertia indices, signature and nullity are calculated using MATLAB [10]. By using "cftoolbox" of MATLAB, a linear polynomial is fitted to the exact values of inertia indices of $G$ and $L(G)$. The obtained data is arranged in Table 1.

\begin{tabular}{|c|c|c|c|c|c|}
\hline$G$ & $p(G)$ & $n(G)$ & $s(G)$ & $\eta(G)$ & $V(G)$ \\
\hline$[m, 1]$ & $6 m$ & $6 m$ & 0 & 0 & $12 m$ \\
\hline$[m, 2]$ & $12 m$ & $12 m$ & 0 & 0 & $24 m$ \\
\hline$[m, 3]$ & $18 m$ & $18 m$ & 0 & 0 & $36 m$ \\
\hline$[m, 4]$ & $24 m$ & $24 m$ & 0 & 0 & $48 m$ \\
\hline
\end{tabular}

\begin{tabular}{|c|c|c|c|c|c|}
\hline$L(G)$ & $p(L(G))$ & $n(L(G))$ & $s(L(G))$ & $\eta(L(G))$ & $V(L(G))$ \\
\hline$[m, 1]$ & $7 m-1$ & $9 m-1$ & 0 & 0 & $12 m$ \\
\hline$[m, 2]$ & $15 m-2$ & $19 m-1$ & 0 & 0 & $24 m$ \\
\hline$[m, 3]$ & $23 m-3$ & $29 m-1$ & 0 & 0 & $36 m$ \\
\hline$[m, 4]$ & $31 m-4$ & $39 m-1$ & 0 & 0 & $48 m$ \\
\hline
\end{tabular}

Table 1: For each curve we have a fixed value of $n$ and for $m=$ $1,2,3, \ldots$ in $\mathrm{V}$-phenylenic and Line of $\mathrm{V}$-phenylenic

The linear curves fitted to the inertia indices, signature and nullity of Vphenylenic $[m, n]$ is shown in Table 1 . Using the data given in this table, a non linear polynomial is fitted. It is noted that the positive and negative inertia are equal for the given nanotube. The inertia of nanotube is plotted using MATLAB as shown in Fig. 3. The results are displayed in Table 2.

The linear curves fitted to the inertia indices, signature and nullity of the line of V-phenylenic $[m, n]$ is shown in Table 1. Using the data in Table 1, the line graph $L(G)$, positive and negative inertia is not equal for numeric value nearest to an integer. The comparison of inertia of the nanotube is plotted using MATLAB as shown in Figs. 4 and 5. The results are displayed in Table 2 . 


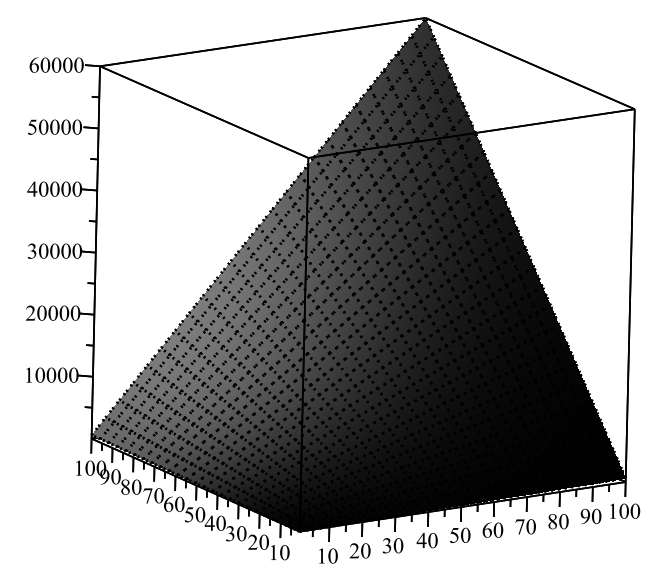

Figure 3: Inertia of $\mathrm{V}$-phenylenic $[m, n]$

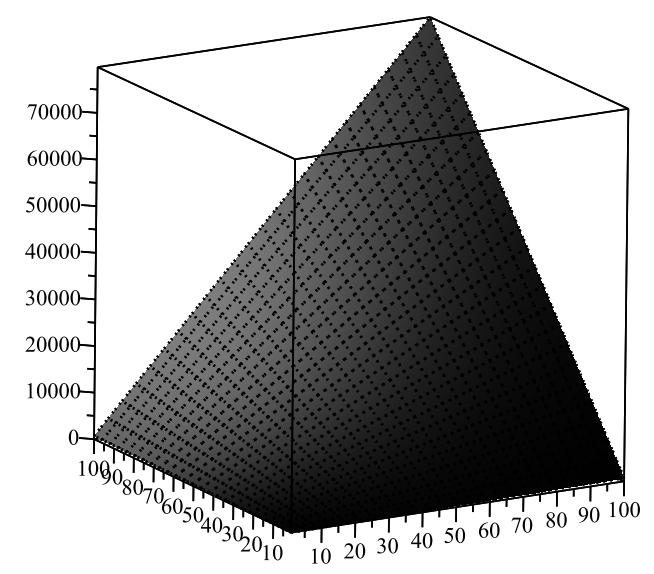

Figure 4: Positive Inertia of Line graph of V-phenylenic $[m, n]$ 


\begin{tabular}{|c|c|c|c|c|c|}
\hline $\mathbf{G}$ & $\mathbf{p}(\mathbf{G})$ & $\mathbf{n}(\mathbf{G})$ & $s(\mathbf{G})$ & $\mathbf{\eta}(\mathbf{G})$ & $\mathbf{V}(\mathbf{G})$ \\
\hline$[\mathrm{m}, \mathrm{n}]$ & $6 \mathrm{mn}$ & $6 \mathrm{mn}$ & $p$ & 0 & $12 \mathrm{mn}$ \\
\hline $\mathbf{L}(\mathrm{G})$ & $\mathbf{p}(\mathrm{L}(\mathbf{G}))$ & $\mathbf{n}(\mathrm{L}(\mathrm{G}))$ & $\mathbf{s}(\mathbf{L}(\mathbf{G}))$ & $\mathbf{\eta}(\mathbf{L}(\mathbf{G}))$ & $\mathbf{V}(\mathbf{L}(\mathbf{G}))$ \\
\hline$[\mathrm{m}, \mathrm{n}]$ & $8 \mathrm{mn}-\mathrm{m}-\mathrm{n}$ & $10 \mathrm{mn}-\mathrm{m}-\mathrm{n}-1$ & $2 \mathrm{mn}-1$ & 1 & $18 \mathrm{mn}-2 \mathrm{~m}-2 \mathrm{n}$ \\
\hline
\end{tabular}

Table 2: For V-phenylenic and Line of V-phenylenic: the non-linear curves fitted of the curves are presented in Table 1

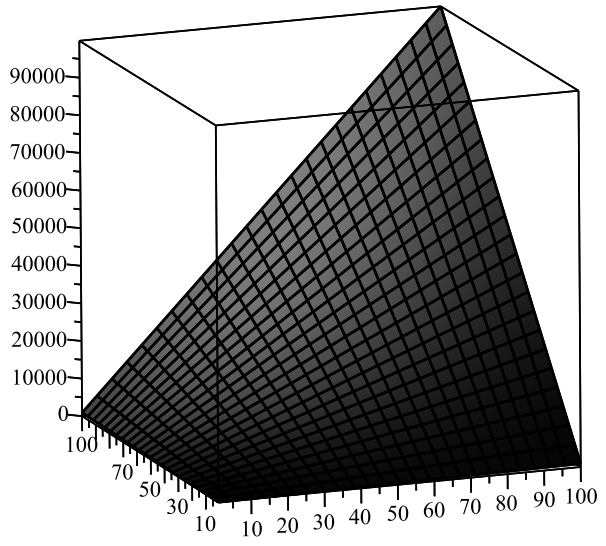

Figure 5: Negative Inertia of Line graph of V-phenylenic $[m, n]$

There are some facts about the given graph and its line graph as follows: Let $G=\mathrm{V}$-phenylenic $[m, n]$ and $L(G)$ is its line graph, then:

- $p(G)=n(G)$,

- $\eta(G)=s(G)=0$,

- $n(L(G))-p(L(G))=s(L(G)))=2 m n-1$,

- $\eta(L(G))=1$.

\section{Conclusion}

In this paper, we have determined that inertia indices, i.e. positive and negative inertia indices are equal for $\mathrm{V}$-phenylenic $[m, n]$ nanotube and these indices 
are not equal for the line graph of $\mathrm{V}$-phenylenic $[m, n]$ nanotube. We also found that the nullity for this nanotube is zero and nullity of line graph of these nanotube is one for integer type data. This means that line graph of V-phenylenic $[m, n]$ nanotube is a singular and V-phenylenic $[m, n]$ nanotube are nonsingular graphs.

\section{Funding}

The authors would like to express their sincere gratitude to the Natural Science Foundation for the Higher Education Institutions of Anhui Province of China (nos. KJ2019A0875 and KJ2019A0876), the Quality Engineering Projects of Anhui Xinhua University of China (no. 2017jxtdx05).

\section{References}

[1] G. Chang, J. Huang, L. Yeh, Hong-Gwa, A characterization of graphs with rank 5, Lin. Alg. and its Appl., 434, No 8 (2011), 1793-1798.

[2] P.W. Atkins, J. de Paula, Physical Chemistry, 8th Ed., Oxford University Press (2006).

[3] M. Bača, J. Horváthová, M. Mokrišová, A. Semaničová-Feňovčiková, A. Suhányiová, On topological indices of carbon nanotube network, Can. J. of Chem., 93, No 10 (2015), 1157-1160.

[4] A.Q. Baig, M. Imran, H. Ali, On topological indices of Poly oxide, Poly silicate, DOX and DSL networks, Can. J. of Chem., 03 (2015); DOI:10.1139/cjc-2014-0490.

[5] S.A.H. Bokhary, M. Imran, S. Manzoor, On molecular topological properties of dendrimers, Can. J. of Chem., 11 (2015); DOI:10.1139/cjc-20150466 .

[6] Chemdraw Software for Windows, PerkinElmer Informatics.

[7] D. Cvetkovic, M. Doob, H. Sachs, Spectra of Graphs: Theory and Application, Academic Press (1980).

[8] M.V. Diudea, O. Ursu, Cs.L. Nagy, Topocluj, Babes-Bolyai University, Cluj (2002). 
[9] M. Imran, A.Q. Baig, H. Ali, On topological properties of dominating David derived networks, Can. J. of Chem., 11 (2015); DOI:10.1139/cjc2015-0185.

[10] MATLAB R2015a, The MathWorks, Inc., Natick, Massachusetts, United States.

[11] N. Trinajstic, Chemical Graph Theory, CRC Press, Boca Raton - FL (1992).

[12] G. Yu, L. Feng, Q. Wang, Bicyclic graphs with small positive index of inertia, Linear Algebra Appl., 438 (2013), 2036-2045.

[13] HyperChem Package Release 7.5 for Windows, Hypercube Inc., Gainesville, Florida 32601, USA (2002). 